\title{
Cambios en el uso de la ostra perlera Pinctada mazatlanica (Bivalvia: Pteriidae) en el Templo Mayor de Tenochtitlan
}

\author{
Changes in the use of the pearl oyster Pinctada mazatlanica (Bivalvia: Pteriidae) in the Great \\ Temple of Tenochtitlan
}

\author{
Adrián Velázquez ${ }^{*}$, Belem Zúñiga-Arellano y John Joseph Temple Sánchez-Gavito² \\ 'Museo del Templo Mayor, Instituto Nacional de Antropología e Historia, Seminario 8, Col. Centro,06060, México D.F. \\ ${ }^{2}$ Centro INAH Baja California, Rayerson 99, Ensenada, 22800 Baja California, México \\ * Correspondencia: adrianveca@yahoo.com
}

\begin{abstract}
Resumen. La concha del bivalvo panámico Pinctada mazatlanica fue ampliamente utilizada por las antiguas sociedades del México prehispánico; en las ofrendas enterradas en el Templo Mayor de Tenochtitlan se han encontrado alrededor de 600 objetos elaborados con ella, la mayor parte de los cuales proceden de la etapa constructiva IV (1440-1481), lo que llama la atención, ya que para entonces los mexicas no habían logrado conquistar emplazamientos en la costa del Pacífico. En los sucesivos agrandamientos arquitectónicos es notable el descenso en los objetos de la referida ostra, lo cual en principio se explicó por la mayor destrucción que éstos habían sufrido; sin embargo, el hallazgo de varios ricos depósitos en el predio conocido como Casa de las Ajaracas, correspondientes al reinado de Moctezuma II (1502-1521), en los que prácticamente se encuentra ausente la Pinctada mazatlanica, da lugar a nuevas interpretaciones. En el presente trabajo se presentan 2 posibles hipótesis para explicar el hecho anterior.
\end{abstract}

Palabras clave: Pinctada mazatlanica, madreperla, Tenochtitlan, sobreexplotación.

\begin{abstract}
The nacreous shell of the tropical Pacific mollusc Pinctada mazatlanica was widely used by the ancient inhabitants of Mexico. Around 600 pieces made of this shell have been found in offerings buried in the Great Temple of Tenochtitlan. Most of these objects come from the IVth construction stage (1440-1481), prior to the conquest of the Pacific Coast by the Aztecs. It was previously thought that the considerably smaller numbers found in the following stages were due to the greater degree of destruction suffered by the temple. Nevertheless, the almost complete absence of this material in nine offerings found recently corresponding to the VIIth construction stage (1502-1521), raise other possibilities. In this work two different hypotheses are presented to explain this observation.
\end{abstract}

Key words: Pinctada mazatlanica, pearl oyster, Tenochtitlan, overexplotation.

\section{Introducción}

La ostra perlera, comúnmente conocida en la región del Golfo de California como madreperla, es un molusco que se distribuye desde la costa externa de Baja California, Golfo de California y hacia el sur hasta Perú; es una especie tropical panámica y bentónica litoral, que depende de un sustrato duro y firme para poder asentarse al final de su vida larvaria y encontrar un hábitat adecuado para su desarrollo. Una de sus características más sobresalientes es poseer un endostraco nacarado (Fig. 1), capaz de producir

Recibido: 13 noviembre 2004; aceptado: 28 marzo 2007 perlas (Keen, 1971), lo que ha llamado la atención de los grupos humanos que habitaron en México desde tiempos antiguos. Se registra su presencia en gran parte del territorio nacional en temporalidades que abarcan desde el periodo Preclásico (1750 - 100 d.C.) hasta la conquista europea (Pires Ferreira, 1978; Mancha, 2002; Velázquez, 1999). Si bien, en las costas fue empleada como recurso alimenticio, en los asentamientos de tierra adentro, su concha fue utilizada para la manufactura de objetos ornamentales, usados por las altas jerarquías sociales.

En las ofrendas del Templo Mayor de Tenochtitlan se ha encontrado una de las mayores concentraciones de objetos manufacturados de Pinctada mazatlanica de la Cuenca 


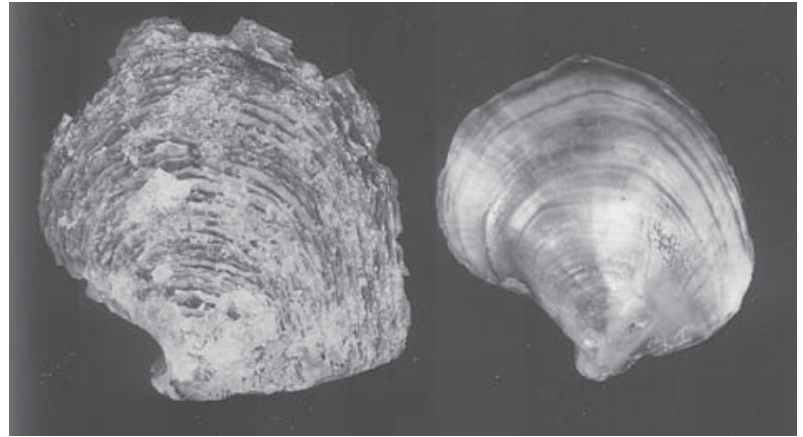

$\mathrm{a}$

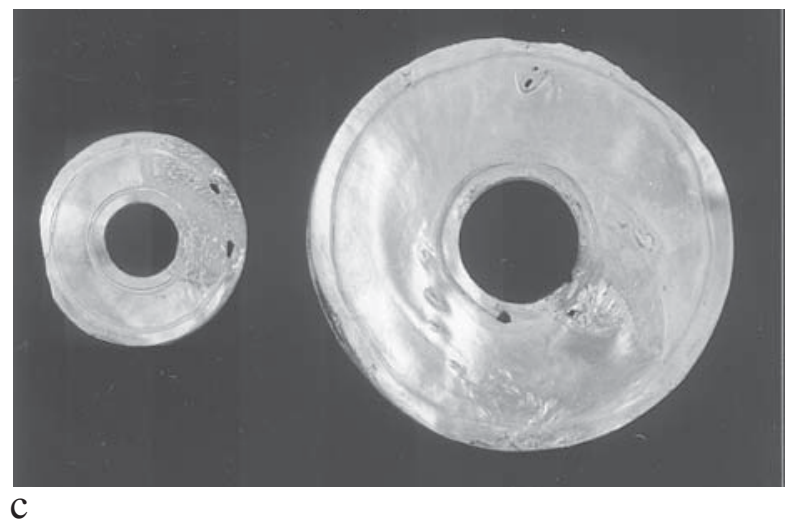

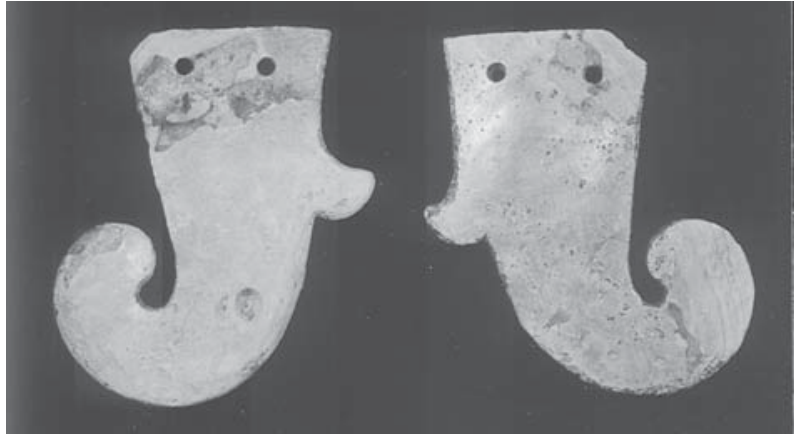

b

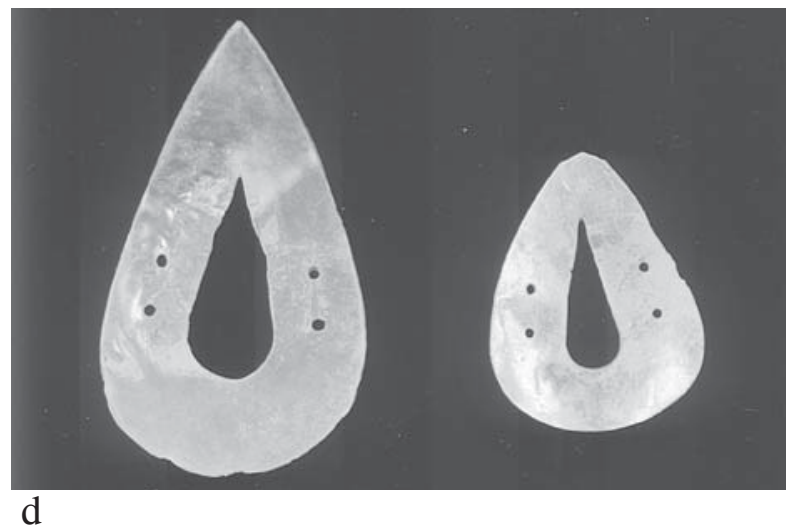

Figura 1. a) Bivalvo Pinctada mazatlanica en estado natural (izq.) y habiéndose eliminado el periostraco y el mesostraco, para dejar al descubierto el endostraco nacarado (der.). Piezas arqueológicas hechas de esta concha, encontradas en las ofrendas del Templo Mayor de Tenochtitlan: orejeras epcololli (b), pectorales anáhuatl (c) y pendientes en forma de gota (d).

de México (595 piezas completas y 605 fragmentos). La importancia del material se pone de manifiesto en el tipo y calidad de las piezas elaboradas, dentro de las que destacan los atributos de importantes deidades, como los conjuntos de pendientes geométricos y zoomorfos de las ofrendas del templo de Tláloc, las orejeras características de Quetzalcóatl y los pectorales de los guerreros estelares, tales como Tezcatlipoca (Velázquez, 1999) (Fig. 1). Es incluso probable que muchos de estos elementos formaran parte central de los principales mensajes plasmados en las oblaciones, como es el caso de la expresión de los ciclos de muerte y resurrección del Sol y Venus (Velázquez, 2000).

Si bien la presencia de una concha panámica no parece extraña en la capital de un imperio que sometió a gran parte del territorio mesoamericano, es interesante que el mayor número de objetos de Pinctada mazatlanica se haya encontrado en las etapas arquitectónicas IVa y IVb, cuya cronología corresponde a los gobiernos de Moctezuma I (1440-1469) y Axayácatl (1469-1481), respectivamente (Matos, 1988), momento en que los mexicas habían sojuzgado a los señoríos del centro de Veracruz; la conquista del litoral Pacífico se haría varios años después, bajo el gobierno de Ahuízotl (1486-1502). Así pues, la obtención de las conchas en cuestión debió darse ya sea a través de las rutas de intercambio que desde tiempos antiguos comunicaban al centro de México con el Pacífico, o bien, obligando a una región que aun cuando no producía directamente dicho recurso, de alguna manera tenía acceso a él (tributación indirecta).

Aparentemente, la disminución en el número de objetos de Pinctada mazatlanica en las etapas arquitectónicas siguientes se debía a la menor conservación de éstas, por la destrucción que sufrió el Templo Mayor después de la

Cuadro 1. Abundancia de objetos de Pinctada mazatlanica por etapa constructiva

\begin{tabular}{ccc}
\hline $\begin{array}{c}\text { Etapa } \\
\text { constructiva }\end{array}$ & $\begin{array}{c}\text { Piezas } \\
\text { completas }\end{array}$ & Fragmentos \\
\hline IV & 572 & 582 \\
V & 3 & 3 \\
VI & 9 & 10 \\
VII & 11 & 10 \\
\hline
\end{tabular}




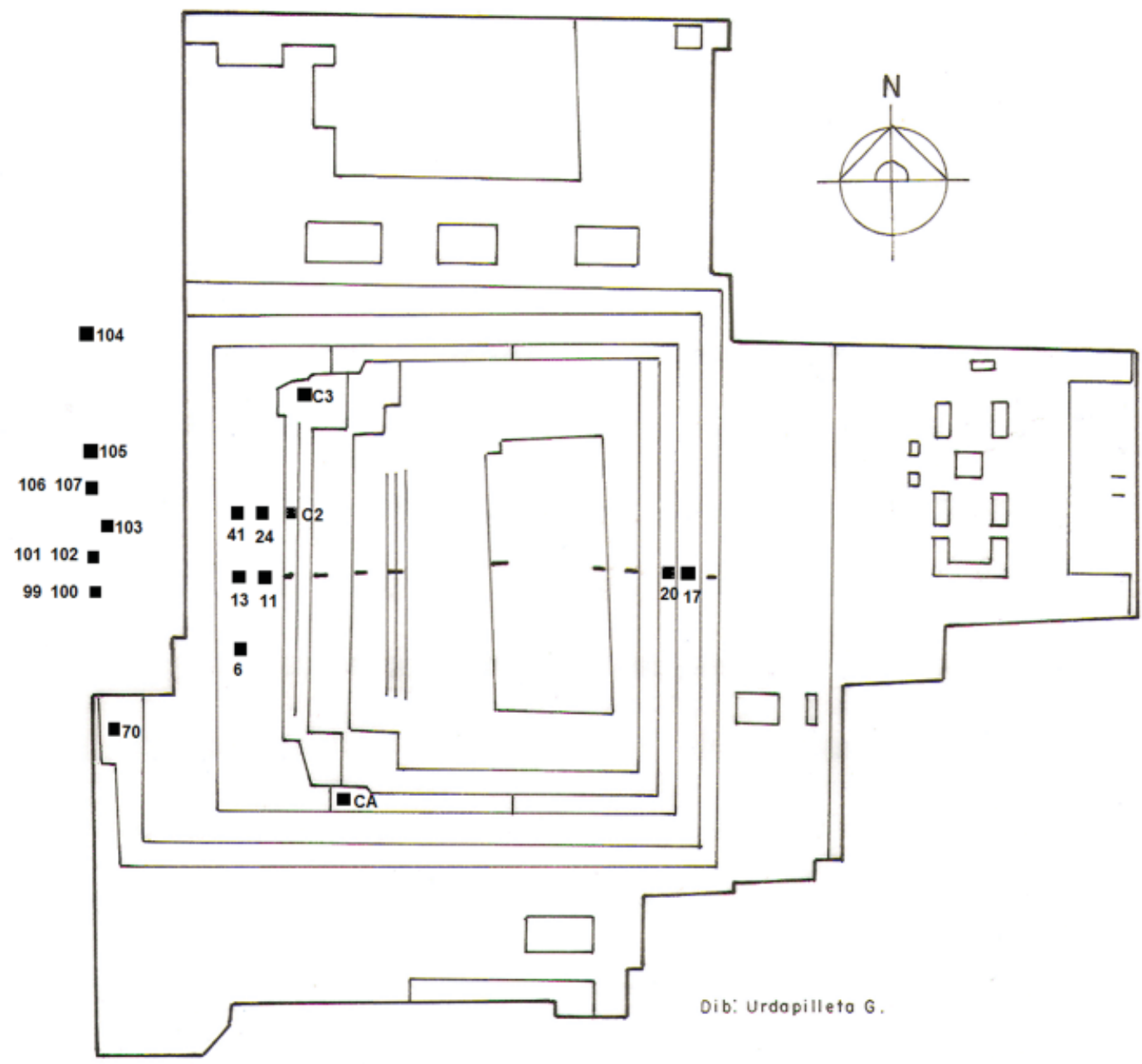

Figura 2. Correspondencia entre las ofrendas de la etapa IV (CA, C2, C3, 6, 11, 13, 17, 20, 24 y 41) en que han sido hallados objetos de Pinctada mazatlanica, y las de la etapa VII $(70,99-107)$, en las que se han encontrado muy pocos.

conquista (Cuadro 1). Sin embargo, el reciente hallazgo de 9 ofrendas en el predio conocido como Casa de las Ajaracas, correspondientes al último agrandamiento del principal edificio de culto de Tenochtitlan, durante el gobierno de Moctezuma II (1502-1520) (Matos, 1988), cambiaría esta visión. Las oblaciones encontradas en dicho lugar tienen correspondencia con varias de las de la etapa IVb de la que proceden muchas de las piezas nacaradas (Fig. 2); por lo que sería de esperarse que hubiera al menos la misma cantidad de este tipo de objetos, ya que para esta época los mexicas tenían pleno dominio de la costa del Pacífico. Sorpresivamente, únicamente fueron halladas 2 valvas de pequeñas dimensiones y ningún objeto manufacturado. En otras ofrendas de la etapa VII, previamente excavadas, se habían encontrado 17 piezas (1 orejera de voluta, 2 pendientes en forma de gota y 14 pectorales circulares incisos y calados).
¿Cómo puede explicarse este marcado descenso en el número de objetos de un material que aproximadamente 50 años antes había sido ampliamente utilizado?

\section{Materiales y métodos}

Con el objetivo de proponer algunas hipótesis a la problemática anteriormente planteada, se consultaron las fuentes documentales del siglo XVI, buscando menciones sobre la explotación de la especie $P$. mazatlanica en el momento del contacto europeo; para ello se consultaron los documentos producto del cuestionario elaborado por la corona española durante el reinado de Felipe II, con la finalidad de lograr un conocimiento profundo de los territorios americanos, conocidos como Relaciones geográficas de Indias, así como las fuentes que describen 
los tributos que recibía Tenochtitlan en la época de Moctezuma II (el Códice Mendocino, la Matrícula de tributos y la Relación de 1554). Previamente, fue necesario identificar el nombre nahua que se daba al mencionado bivalvo, para lo que fue de ayuda invaluable el libro undécimo del Códice florentino en el que se consultó la versión paleografiada y traducida del náhuatl por López (1991).

\section{Resultados}

Los antiguos nahuas empleaban una diversidad de nombres para referirse a distintos tipos de moluscos. Así, el nombre atzcalli era un genérico de almeja (López, 1991), al cual se le agregaban otras palabras para describir características específicas; en el Códice florentino se dan diferentes nombres a las conchas nacaradas: quetzalatzcalli (concha preciosa), chalchiuhatzcalli (concha de jade) uitzitzilatzcalli (concha tornasolada como el colibrí). En la misma fuente se describen estas conchas como de apariencia tosca y burda por fuera, con un interior bruñido y liso, admirable y maravilloso.

“...De él se generan completamente los colores: rojo, amarillo [es como el] xiuhtótotl, [es como el] tlauhquéchol, verde, violeta, rojizo, color denso, rosado; allí están todos [los colores] reunidos. Su apariencia es algunas veces como el arcoiris. Es algunas veces como el brillo de la luciérnaga. Es de diversas pinturas, de diversos colores, muy fraccionado, muy partido" (López, 1991).

Otro nombre que se daba a las conchas nacaradas era el de eptli, donde la partícula ep indica la apariencia tornasolada; de hecho a las perlas se les denominada epyollotli (corazón de concha) (López, 1991).

Las almejas de tonalidades rojizas que en su superficie criaban diversos organismos marinos recibían el nombre de tapachtli, y correspondían a especies de las familias Chamidae y Spondylidae (Temple y Velázquez, 2003).

Al revisar las fuentes históricas del siglo XVI llama poderosamente la atención la total ausencia en los registros de la tributación de las almejas nacaradas marinas. En la lámina 18 de la Matrícula de tributos (1991) se representan 2 valvas rojas y espinosas, glosadas como tapachtli, que en su correspondiente del Códice Mendocino (1979) se describen como "conchas de la mar como veneras coloradas"; tanto por su nombre en náhuatl (tapachtli) como por su representación, parecen corresponder a especímenes de Spondylus princeps; éstos eran tributados semestralmente por varios pueblos de la costa del Pacífico, que quizás se distribuyeran desde Colima hasta el actual Acapulco (Temple y Velázquez, 2003). Sin embargo, en ninguna ocasión se habla de ejemplares nacarados. La misma situación se encontró en la Información de 1554, con la diferencia de que se refiere al tapachtli como piedras rojas para hacer máscaras.

Por su parte, las Relaciones geográficas del siglo $X V I$ correspondientes a las poblaciones asentadas en las inmediaciones del litoral del Pacífico, en ningún momento mencionan la explotación de la madreperla, lo cual seguramente no hubiera sido un dato que dejaran pasar los españoles, siempre ávidos de cualquier fuente de riqueza. Las únicas referencias al respecto las proporcionan las relaciones de la Villa de la Purificación y la de la Provincia de Motines; en el primer caso, los habitantes de Cuamichitlan y Chametla, poblaciones cercanas a Mazatlán, reportan que a sus gobernadores "les tributaban mantas, perlas, gallinas y plata que había de sus tratos y contratos que tenían" (Gómez de Alvarado, 1988); en el segundo, los nativos de Huiztlan, situada en las inmediaciones de los límites de los actuales estados de Guerrero y Michoacán, narran que "antiguamente, solían sacar los indios deste pueblo aljófar y perlas de la mar, y ahora no, porque dicen que son muertos los que lo sabían sacar" (Alcalde de Rueda, 1985).

\section{Discusión}

A partir de la información de las fuentes documentales del siglo XVI, puede deducirse que para esta época no existía una explotación importante de la $P$. mazatlanica, en la zona del litoral del Pacífico que estuvo bajo el control del imperio azteca; los documentos únicamente hacen mención de la tributación del tapachtli. Al respecto podemos proponer 2 explicaciones:

Baja poblacional por sobreexplotación del recurso. Como anteriormente se mencionó, la $P$. mazatlanica fue una almeja ampliamente utilizada desde tiempos muy antiguos. Para el periodo Formativo es la concha que se presenta de forma más abundante en los registros arqueológicos de los sitos de tierra adentro, situación que se incrementa para el Clásico. Para el Posclásico en la cuenca de México el gusto por dicho material queda evidenciado en su gran abundancia en las ofrendas del Templo Mayor. ¿Podría ser que una explotación intensiva por un prolongado periodo de tiempo resultara a la postre en una extinción de los bancos accesibles a las técnicas de recolección de las sociedades prehispánicas?

Aun cuando la distribución de la especie abarca desde el Golfo de California hasta Ecuador, los registros de colectas actuales son escasos en la zona en la que potencialmente los mexicas pudieron haberla obtenido. Esta misma situación parece haberse presentado en la época prehispánica (Zúñiga, 2003), puesto que en varios 
asentamientos costeros de dicha área, cuya cronología abarca desde el Preclásico hasta el contacto europeo, no se han identificado conchas de $P$. mazatlanica. Si bien esto puede explicarse por el alto valor del recurso, causante de la exportación de las madreperlas a los sitios de tierra adentro; también es necesario tomar en cuenta que en las provincias malacológicas los moluscos no se encuentran de forma continua, habiendo zonas de mayor concentración, que se alternan con otras de menor o incluso nula presencia.

Quizás en las zonas de obtención de P. mazatlanica para las sociedades del Centro de México los bancos no eran naturalmente muy abundantes, ocasionando la explotación sistemática de ellos por un prolongado periodo de tiempo, una merma considerable de la que la población difícilmente se recuperaría. Debe llamarse la atención sobre el hecho de que únicamente asentamientos tan norteños como Cuamichitlan y Chametla, cercanos al actual Mazatlán y fuera del área de influencia de los mexicas, registran la pesquería de las perlas para el siglo XVI.

Cambio de preferencia cultural. Otra posibilidad para explicar el drástico descenso en el número de objetos nacarados en las ofrendas del Templo Mayor posteriores a la etapa IV, podría ser un cambio en la preferencia por determinados materiales. Un buen ejemplo de ello lo representa el disco de turquesa que apareció en la ofrenda 99-una de las oblaciones localizadas en el predio de la Casa de las Ajaracas- , en el cual se representa a Huitzilopochtli junto con Venus y otros guerreros estelares durante el viaje que se pensaba llevaban a cabo por el inframundo durante la noche (Velázquez y Marín, 2002). Este mismo ciclo es expresado en las ofrendas de la etapa IVb a través de conjuntos de objetos, en los que las piezas de concha-entre ellos importantes atributos hechos de $P$. mazatlanicajugaban un papel preponderante (Velázquez, 2000).

Resulta sugerente que la etapa IV del Templo Mayor corresponda con el Xiuhmolpilli de 1455, fiesta que conmemoraba el fin de un siglo mesoamericano, momento de gran peligro ante el riesgo de que el Sol no volviera a nacer, lo que traería como consecuencia el desplome de la bóveda celeste y el fin del mundo y de la humanidad presentes. Es asimismo interesante que dicha ceremonia fue la primera que celebraron los mexicas después de liberarse del yugo de los tepanecas de Azcapotzalco y tras haber iniciado su carrera imperial. ¿Podría ser que en las oblaciones de dicha ampliación arquitectónica se representara la muerte del cuarto Sol, que era Quetzalcóatl, expresada a través de objetos de concha, material perteneciente al ámbito del inframundo y por lo tanto del pasado? Es de sobra sabido que el pueblo azteca al tomar preponderancia en el escenario político proclamó el nacimiento de una nueva era y por lo tanto de un nuevo Sol, Huitzilopochtli (Graulich, 1990). Debe hacerse notar el hecho de que el ciclo de muerte y resurrección del astro se encuentre prácticamente confundido con el de Venus en las ofrendas de la etapa IV, manifestación astral de Quetzalcóatl (Velázquez, 2000).

Por su parte, en la ofrenda 99, depositada en la época de máximo esplendor, aparece una pieza hecha de un material ígneo, ya que el nombre nahua de la turquesa, xíhuitl, alude también al fuego, al año y a la hierba. En ella aparece la imagen de Huitzilopochtli, el dios solar y tutelar de los mexicas, acompañado por los guerreros estelares durante su viaje por el interior de la tierra; quizás la ausencia de atributos de concha señale la existencia del sol de los mexicas o quinto Sol.

La información recopilada hasta el momento no permite inclinar la balanza por ninguna de las posibilidades planteadas. Por una parte, sí parece haber habido un descenso poblacional importante en los bancos de $P$. mazatlanica de la zona del Pacífico controlada por los mexicas, al grado que su explotación no está registrada en ninguna de las fuentes documentales del siglo XVI. Por la otra, no parece de ninguna manera casual la sustitución de materiales que se da para expresar ciclos cosmogónicos similares, entre las etapas IV y VII. Quizás el descenso de un recurso tan preciado fuera interpretado como una manifestación del fin de una era para el inicio de otra.

\section{Literatura citada}

Alcalde de Rueda, J. 1985. Relación de Motines. In Relaciones y memorias de la provincia de Michoacán 1579-1581, A. Ochoa y G. Sánchez (eds.). Universidad Michoacana y Ayuntamiento de Morelia, Michoacán. p. 157-180.

Códice Mendocino. 1979. San Ángel, México, D.F. 194 p.

Gómez de Alvarado, L. 1988. Relación de la Villa de Purificación (19 de enero de 1585). In Relaciones geográficas del siglo XVI: Nueva Galicia, tomo 10, R. Acuña (ed.). Universidad Nacional Autónoma de México, México, D.F. p. 209-235.

Graulich, M. 1990. Mitos y rituales del México antiguo. Istmo, Madrid. 503 p.

Información de 1554, sobre los tributos que los indios pagaban a Moctezuma. 1997. J. L. de Rojas (edición y paleografía). CIESAS, México, D.F. 208 p.

Keen, M. 1971. Sea shells of tropical west America. Stanford University Press, California. 1064 p.

López Luján L. 1991. Peces y moluscos en el libro undécimo del Códice florentino. In $\mathrm{La}$ fauna en el Templo Mayor, O. J. Polaco (coord.). Instituto Nacional de Antropología e Historia, GV editores y Asociación de Amigos del Templo Mayor, México, 
D. F. p. 213-263.

Mancha, E. 2002. Objetos de concha en contextos arqueológicos de la cuenca de México en la época prehispánica. Tesis, Escuela Nacional de Antropología e Historia, México. 535 p.

Matos, E. 1988. The Great Temple of the Aztecs. Thames and Hudson, London. 192 p.

Matrícula de tributos. 1991. Secretaría de Hacienda y Crédito Público, México, D. F. 154 p.

Pires-Ferreira, J. W. 1978. Shell exchange networks in Formative Mesoamerica. In Cultural continuity in Mesoamerica, D. L. Browman (ed.). Mouton, The Hague. p. 79-100.

Temple, J. J. y A. Velázquez. 2003. El tapachtli entre los antiguos nahuas. In Estudios etnobiológicos, pasado y presente de México, A. Motúfar López (coord.). Instituto Nacional de Antropología e Historia, México, D. F. p. 13-23.
Velázquez, A. 1999. Tipología de los objetos de concha del Templo Mayor de Tenochtitlan. Colección Científica 392. Instituto Nacional de Antropología e Historia, México, D.F. 134 p.

Velázquez, A. 2000. El simbolismo de los objetos de concha encontrados en las ofrendas del Templo Mayor de Tenochtitlan. Colección Científica 403. Instituto Nacional de Antropología e Historia, México, D. F. $322 \mathrm{p}$.

Velázquez, A. y M. E. Marín. 2002. The turquoise tile disc of the offering 99 of the Great Temple of Tenochtitlan. $67^{\text {th }}$ Annual Meeting, Society for American Archaeology, Denver, Colorado.

Zúñiga, B. 2003. Moluscos arqueológicos de Ixtapa Cihuatanejo- Petatlán, Guerrero. In Estudios etnobiológicos, pasado y presente de México. A. Motúfar López (coord.) Instituto Nacional de Antropología e Historia, México, D. F. p. 39-60. 